muscular dystrophy in association with malignant hyperthermia or anesthetic induced cardiac arrest. This patient had neither myoglobinuria nor hyperthermia. A neonatal presentation of Duchenne muscular dystrophy is exceptional, signs usually appearing when the patient becomes ambulant.

NEONATAL GUILLAIN-BARRE' SYNDROME

A term female infant presenting with generalized hypotonia, paucity of lower limb movements, and diminished DTRs was diagnosed as a case of Guillain-Barre' syndrome at the Hospital for Sick Children, University of Toronto, Canada. At 3 weeks of age, motor nerve conduction studies showed slowed velocities and decreased action potentials, and the CSF protein was elevated with normal cells. Clinical improvement began at age 2 weeks and examination was normal at 22 weeks. (Al-Qudah AA et al. Neonatal Guillain-Barre' syndrome. Pediatr Neurol Aug $1988 ; \underline{4}: 255-6$ ).

\title{
NEONATAL MYASTHENIA GRAVIS
}

The advantages of electrodiagnosis in a premature infant with neonatal myasthenia gravis are proposed by the Dept of Rehabilitation Medicine, Children's Hospital and Medical Center, Univ of Washington School of Medicine, Seattle, WA. The infant, born to a mother with myasthenia, suffered hypoxia and subependymal hemorrhage which probably contributed to the hypotonia and poor respiratory effort. Testing with edrophonium, $0.1 \mathrm{mg} / \mathrm{kg}$ IV demonstrated no clinical improvement, whereas repetitive motor nerve stimulation testing showed a significant decremental response consistent with a diagnosis of neonatal myasthenia gravis. The decremental response was corrected following IV infusion of edrophonium $0.15 \mathrm{mg} / \mathrm{kg}$. Pyridostigmine in a dose of $8 \mathrm{mg} / \mathrm{kg} /$ day resulted in clinical improvement of respiratory and muscular activity, and the infant was weaned from assisted ventilation at 27 days of age. He was discharged at 41 days of age on pyridostigmine therapy. The authors conclude that repetitive motor nerve stimulation may be a more reliable diagnostic procedure than edrophonium IV in the newborn with suspected myasthenia gravis. (Hays RM, Michaud LJ. Neonatal myasthenia gravis: Specific advantages of repetitive stimulation over edrophonium testing. Pediatr Neurol Aug $1988 ; \underline{4}: 245-7$ ).

COMMENT. The value of electrodiagnostic tests in the differential diagnosis of the hypotonic infant is demonstrated in these 2 case reports. Neonatology texts often recommend edrophonium as the test of choice in neonatal myasthenia gravis. The above experience indicates that the pharmacological test alone may not be as sensitive as repetitive nerve stimulation in the newborn with multiple problems. Ultrasonography is an additional technique of potential value in the work up of the hypotonic infant. Heckmatt $\mathrm{Jz}$ and Dubowitz $\mathrm{V}$ of 
Hammersmith Hospital, London, have introduced the method for the differentiation of congenital muscular dystrophy and non-neuromuscular cases of hypotonia. ( $\mathrm{J}$ Child Neurol $1987 ; \underline{2}$ : 205$)$.

\title{
CONGENITAL MYOTONIC DYSTROPHY AND PLEURAL EFFUSION
}

Two infants with congenital myotonic dystrophy complicated by pleural effusions and hydrops fetalis are reported from the Valley Children's Hospital, Fresno, CA, and the Royal Alexandra Hospitals, University of Alberta, Edmonton, Alberta, Canada. The mothers had myotonic dystrophy diagnosed at or after the delivery. The pregnancies were complicated by polyhydramnios, and the infants were delivered by cesarean section because of breech presentation. Infant 1 was areflexic, profoundly hypotonic, failed to breathe spontaneously, and died at 3 days of age. Muscle biopsy at autopsy revealed extreme muscle immaturity with poor type I/II fiber differentiation, type I and type IIc fibers, and central nucleation. Infant 2 died 25 min after delivery despite aggressive resuscitation. The authors cite 6 other cases of congenital myotonic dystrophy with fetal hydrops and 2 with pleural effusions. Fetal hydrops may obscure the diagnosis, especially if the mother is asymptomatic. (Curr CJR et al. Hydrops and pleural effusions in congenital myotonic dystrophy. J Pediatr Sept 1988; $113: 555-557$ ).

\begin{abstract}
COMMENT. Chromosomal defects, cardiac abnormalities, and genetic syndromes are described with fetal hydrops but congenital myotonic dystrophy has been associated infrequently. When a mother is known to have myotonic dystrophy, the fetus should be monitored for abnormal breathing patterns and pleural effusions. A hypotonic infant born with pleural effusion or hydrops should alert the examiner to check for myotonia in the mother. (See Ped Neur Briefs Sept $1987 ; \underline{1}: 29-30)$.
\end{abstract}

\section{NEONATAL CEREBRO-VASCULAR DISORDERS}

\section{NEONATAL STROKE OUTCOME}

The clinical outcome of 17 children, 1 to 11 years of age, who experienced cerebral artery infarctions as neonates has been studied in the Depts of Pediatrics and Neurology, Univ of Kentucky Med Center, Lexington, Kentucky. The left middle cerebral artery (MCA) was involved in 9 (53\%) and the right MCA in $5(30 \%)$. Fourteen $(82 \%)$ who developed neonatal seizures became seizure free and neurologically normal within the first year and anticonvulsants were discontinued. Three patients had recurrence of seizures after 1 to 8 years and anticonvulsants were renewed. Eleven patients (65\%) have normal neurologic development but one of 2 attending school has cognitive 